\title{
Lactiplantibacillus plantarum Reduced Renal Calcium Oxalate Stones by Regulating Arginine Metabolism in Gut Microbiota
}

OPEN ACCESS

Edited by:

Gian/uigi Mauriello,

Università degli Studi di Napoli

Federico II, Italy

Reviewed by:

Zhangqun Ye,

Huazhong University of Science

and Technology, China

Zhaolai Dai,

China Agricultural University, China

*Correspondence:

Kunjie Wang

wangkj@scu.edu.cn

Qun Sun

qunsun@scu.edu.cn

tThese authors have contributed equally to this work and share first authorship

Specialty section: This article was submitted to

Food Microbiology,

a section of the journal

Frontiers in Microbiology

Received: 17 July 2021 Accepted: 02 September 2021 Published: 24 September 2021

Citation:

Liu Y, Jin X, Tian L, Jian Z, Ma Y, Cheng L, Cui Y, Li H, Sun $Q$ and

Wang K (2021) Lactiplantibacillus plantarum Reduced Renal Calcium Oxalate Stones by Regulating Arginine

Metabolism in Gut Microbiota.

Front. Microbiol. 12:743097.

doi: 10.3389/fmicb.2021.743097

\author{
Yu Liu't, Xi Jin't, Lei Tian'2, Zhongyu Jian', Yucheng Ma', Liang Cheng1, Yaqian Cui', \\ Hong Li', Qun Sun ${ }^{2 *}$ and Kunjie Wang ${ }^{1 *}$
}

\footnotetext{
1 Laboratory of Reconstructive Urology, Department of Urology, Institute of Urology, West China Hospital, Sichuan University, Chengdu, China, ${ }^{2}$ Key Laboratory of Bio-Resources and Eco-Environment of Ministry of Education, College of Life Sciences, Sichuan University, Chengdu, China
}

Renal calcium oxalate (CaOx) stones are a common kidney disease. There are few methods for reducing the formation of these stones. However, the potential of probiotics for reducing renal stones has received increasing interest. We previously isolated a strain of Lactiplantibacillus plantarum N-1 from traditional cheese in China. This study aimed to investigate the effects of $\mathrm{N}-1$ on renal $\mathrm{CaOx}$ crystal deposition. Thirty rats were randomly allocated to three groups: control group ( $\mathrm{ddH} 2 \mathrm{O}$ by gavage), model group [ddH2O by gavage and $1 \%$ ethylene glycol $(E G)$ in drinking water], and Lactiplantibacillus group ( $\mathrm{N}-1$ by gavage and 1\% EG in drinking water). After 4 weeks, compared with the model group, the group treated with $\mathrm{N}-1$ exhibited significantly reduced renal crystals $(P<0.05)$. In the ileum and caecum, the relative abundances of Lactobacillus and Eubacterium ventriosum were higher in the control group, and those of Ruminococcaceae UCG 007 and Rikenellaceae RC9 were higher in the $\mathrm{N}$-1-supplemented group. In contrast, the relative abundances of Staphylococcus, Corynebacterium 1, Jeotgalicoccus, Psychrobacter, and Aerococcus were higher in the model group. We also predicted that the arginase level would be higher in the ileal microbiota of the model group than in the N-1-supplemented group with PICRUSt2. The arginase activity was higher, while the level of arginine was lower in the ileal contents of the model group than in the $\mathrm{N}$-1-supplemented group. The arginine level in the blood was also higher in the $\mathrm{N}$-1-supplemented group than in the model group. In vitro studies showed that exposure to arginine could reduce $\mathrm{CaOx}$ crystal adhesion to renal epithelial $\mathrm{HK}-2$ cells. Our findings highlighted the important role of $\mathrm{N}-1$ in reducing renal $\mathrm{CaOx}$ crystals by regulating arginine metabolism in the gut microbiota. Probiotics containing L. plantarum N-1 may be potential therapies for preventing renal $\mathrm{CaOx}$ stones.

Keywords: renal stones, calcium oxalate, Lactiplantibacillus plantarum, gut microbiota, arginine 


\section{INTRODUCTION}

Renal stones are a common kidney disease worldwide that can cause pain, urinary tract infection, chronic renal disease, or even loss of renal function. The prevalence of these stones has been increasing, being approximately $6.4 \%$ in China (Zeng et al., 2017), 6.3\% in the United States (Tundo et al., 2018), and 5\% in Europe (Sorokin et al., 2017). Treatments for renal stones include drugs, extracorporeal shock wave lithotripsy, flexible ureteroscopic lithotripsy, and percutaneous nephrolithotomy. Although most renal stones can be removed successfully using these therapies, the recurrence rate is high at $53 \%$ after 5 years (Liu et al., 2018). The annual cost of renal stone disease in 2000 in the United States was approximately $\$ 2.81$ billion and will continue to rise (Antonelli et al., 2014).

The main component of renal stones is calcium oxalate $(\mathrm{CaOx})$, which accounts for approximately $80 \%$ of renal stones (Knoll et al., 2011). Only a few renal $\mathrm{CaOx}$ stones are caused by primary hyperoxaluria due to the lack of enzymes alanine-glyoxylate-aminotransferase or glyoxylate reductase/hydroxypyruvate reductase in the liver (Hoppe, 2012). Some renal $\mathrm{CaOx}$ stone patients are concurrently diagnosed with hyperparathyroidism, resulting in excessive parathyroid hormone secretion and hypercalciuria, followed by renal stones (Minisola et al., 2018). However, the reason for renal $\mathrm{CaOx}$ stones in the majority of patients is still unknown.

Oxalate and its precursors in food are absorbed into the blood, and then excreted into the urine (Sadaf et al., 2017). Recently, some studies have focused on the association between gut microbiota and renal $\mathrm{CaOx}$ stones. It was reported that Oxalobacter formigenes could degrade oxalate into formate through formyl-CoA transferase and oxalyl-CoA decarboxylase (Sadaf et al., 2017). The urinary oxalate level decreased in rats with hyperoxaluria after supplementation with $O$. formigenes (Sidhu et al., 2001; Hatch et al., 2011). However, the results of clinical trials were contrary to each other. A randomised phase II/III study reported that the changes in urinary oxalate levels were not different between primary hyperoxaluria patients treated with or without O. formigenes (Milliner et al., 2018). Another ongoing Phase II study found that 24 months O. formigenes administration substantially decreased plasma oxalate concentrations when compared with an untreated natural control cohort (Hoppe et al., 2021).

In addition, probiotics containing Lactobacillus and Bifidobacterium have demonstrated significant potential for treating various diseases. It was reported that the intake of probiotics could control oxidative stress and systemic inflammation in chronic kidney disease patients (Lopes et al., 2018). Some studies found that the administration of lactic acid bacteria reduced urinary oxalate levels in nephrolithiasis patients (Campieri et al., 2001; Lieske et al., 2005). However, several studies reported the different results that lactic acid bacteria neither reduced urinary oxalate excretion nor plasma oxalate concentration (Goldfarb et al., 2007; Siener et al., 2013). A probiotic supplement $\left(\mathrm{VSL} \# 3^{\circledR}\right)$, containing Streptococcus thermophilus, Bifidobacterium, and Lactobacillus, could reduce gastrointestinal oxalate absorption (Okombo and Liebman, 2010;
Al-Wahsh et al., 2012). These evidences indicated that the supplementation with probiotics may be a potential strategy for preventing renal stones, which need further studies. We previously isolated seven strains of Lactiplantibacillus plantarum from two kinds of traditional cheeses in Daocheng, Sichuan, China (Liu et al., 2017), which were safe to use as probiotics. L. plantarum K41 could inhibit the formation of Streptococcus mutans biofilms. The aim of the current study was to explore whether one of the strains, $L$. plantarum N-1, could reduce the formation of renal crystals and to study the role of gut microbiota during this process.

\section{MATERIALS AND METHODS}

\section{Culture of Lactiplantibacillus plantarum $\mathrm{N}-1$}

The L. plantarum N-1 strain preserved in glycerin was obtained from the Key Laboratory of Bioresources and Eco-environment of The Ministry of Education, Sichuan University. N-1 strains were cultured in $10 \mathrm{~mL}$ MRS medium (Difco, China) at an inoculation of $1 \%(\mathrm{v} / \mathrm{v})$ for $18 \mathrm{~h}$ at $37^{\circ} \mathrm{C}$. Then, the suspension of strains was centrifuged for $5 \mathrm{~min}$ at $3,000 \times \mathrm{g}$. After two washes, the strains were resuspended in $10 \mathrm{~mL}$ saline solution. The bacterial suspension was adjusted to $10^{8} \mathrm{CFU} / \mathrm{mL}$ with a spectrophotometer and preserved in a centrifuge tube at $4^{\circ} \mathrm{C}$ for further use.

\section{Development of Rats With Renal Calcium Oxalate Stones and Administration of Lactiplantibacillus plantarum N-1}

We purchased 6-week-old male Sprague-Dawley rats from Dossy Experimental Animals Co., Ltd. (Chengdu, Sichuan, China). The mean body weight of rats was $198 \mathrm{~g}$. The rats were first acclimatised for 1 week before the experiment in the specificpathogen-free facility at the Animal Experiment Center of West China Hospital, Sichuan University. The rats were given standard food and sterile water. We obtained ethical approval from the West China Hospital of Sichuan University Medical Research Ethics Committee (protocol numbers: 2017063A).

Thirty rats were randomly allocated to three groups: the control group, model group, and Lactiplantibacillus group. The

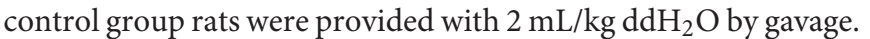
Apart from $\mathrm{ddH}_{2} \mathrm{O}$ gavage, the rats in the model group had free access to drinking water with $1 \%(\mathrm{v} / \mathrm{v})$ ethylene glycol (EG). In the Lactiplantibacillus group, the rats had drinking water with $1 \%$ EG and a suspension of $\mathrm{N}-1\left(1 \times 10^{8} \mathrm{CFU} / \mathrm{mL}, 2 \mathrm{~mL} / \mathrm{kg}\right)$ by gavage once a day. After 4 weeks, $24 \mathrm{~h}$ urine of all the rats was collected using metabolic cages. Each urine sample was divided into three aliquots, which were supplemented with $0.5 \mathrm{~mL}$ hydrochloric acid $(6 \mathrm{~mol} / \mathrm{L}) / 10 \mathrm{~mL}$ urine to prevent the degradation of oxalate (Wu et al., 2015). Then, we injected chloral hydrate [4\% (w/v), $0.8 \mathrm{~mL} / 100 \mathrm{~g}$ ] intraperitoneally to euthanise the rats. Blood was drawn from the heart and centrifuged for $10 \min \left(1,500 \times g, 4^{\circ} \mathrm{C}\right)$ to obtain plasma. Renal tissues were fixed in $10 \%$ formaldehyde and embedded in paraffin. The remaining kidneys, plasma 
and intestinal contents in the ileum, caecum, and colon were stored at $-80^{\circ} \mathrm{C}$.

\section{Observation of Renal Crystals and Urinary Oxalate}

Haematoxylin-eosin (HE) and von Kossa (VK) staining methods were used to observe the renal $\mathrm{CaOx}$ crystals. First, kidney sections $(3-4 \mu \mathrm{m})$ were prepared and stained with HE following the manufacturer's protocols for the staining kit (Thermo Fisher Scientific, United States). Second, VK staining was also performed to detect calcium crystals using a staining kit (Solarbio, Beijing, China). We analysed the staining images using a microscope (magnification, $\times 100$; CARL ZEISS, Heidenheim, Germany). The HE staining scoring system reported by Xiang et al. (2015) and the VK staining scoring method using ImagePro Plus 6 software were separately applied as semiquantitative and quantitative methods to evaluate renal crystals. Higher scores indicated more crystal deposition.

Urinary oxalate was measured using liquid chromatographymass spectrometry. Briefly, $10 \mu \mathrm{L}$ hydrochloric acid $(12 \mathrm{~mol} / \mathrm{L})$ and $200 \mu \mathrm{L}$ 1,2-diaminobenzene $(5 \mathrm{mg} / \mathrm{mL})$ were added to the $100 \mu \mathrm{L}$ urine, which was then heated at $140^{\circ} \mathrm{C}$ for $40 \mathrm{~min}$. When the sample was cooled to room temperature, it was added with $10 \mu \mathrm{L}$ sodium hydroxide $(10 \mathrm{~mol} / \mathrm{L})$ and centrifuged for $10 \mathrm{~min}$ $(1,500 \times g)$. Then, $100 \mu \mathrm{L}$ supernatant was added with $300 \mu \mathrm{L}$ acetonitrile and centrifuged for $10 \mathrm{~min}(1,500 \times g)$. The oxalate was turned into 2,3-dihydroxyquinoxaline. The samples were examined using an LCMS-8040 system (Shimadzu, Kyoto, Japan).

\section{Examination of Gut Microbiota}

The 16S rRNA gene sequencing technique was applied to analyse the gut microbiota. Total microbial DNA was extracted from faecal samples. Then, the V3-V4 regions of the $16 \mathrm{~S}$ rRNA gene were amplified with polymerase chain reaction. The amplification products were sequenced on an Illumina MiSeq platform (Illumina, San Diego, CA, United States). The sequences were classified into different operational taxonomic units (OTUs) when their similarity was $97 \%$ or higher. The Silva 16 S rRNA database (version 138) was used to identify the taxonomy of the OTUs.

\section{Determination of Amino Acids in the lleal Contents and Blood}

Amino acids were measured by liquid chromatography-mass spectrometry. We mixed $100 \mathrm{mg}$ of ileal contents with $1 \mathrm{~mL}$ acetonitrile/methanol/water (2:2:1, v/v) and sonicated them for $30 \mathrm{~min}\left(4^{\circ} \mathrm{C}\right)$, followed by centrifugation for $20 \mathrm{~min}(15,000 \times \mathrm{g}$, $\left.4^{\circ} \mathrm{C}\right)$. Fifty microlitres of plasma and $200 \mu \mathrm{L}$ of methanol acetonitrile/methanol $(1: 1, \mathrm{v} / \mathrm{v})$ were mixed. We left the mixture to stand for $60 \mathrm{~min}$ before centrifugation for $20 \mathrm{~min}(15,000 \times \mathrm{g}$, $4^{\circ} \mathrm{C}$ ). Then, we applied the supernatant to UHPLC (Agilent, Santa Clara, CA, United States) and a QTRAP 5500 system (Sciex, Redwood, VA, United States). If the relative standard deviations of quality control (containing the same amount of contents from each sample) for all amino acids were less than $30 \%$, the measurements were reproducible and stable. The information of amino acids standards used for liquid chromatography-mass spectrometry analysis was shown in Supplementary Table 1. The relative quantitative data for each amino acid was calculated based on the peak areas and retention times obtained from Multiquant software. The standards were used to calculate the retention time and identify amino acids.

\section{Measurement of Arginase Activity in the Ileal Contents}

Arginase activity was determined using a colorimetric arginase activity assay kit (ab180877, Abcam, Cambridge, United Kingdom) according to the manufacturer's protocol. Briefly, each sample well was added with $20 \mu \mathrm{L}$ plasma, $20 \mu \mathrm{L}$ assay buffer and $10 \mu \mathrm{L}$ substrate mix. Each standard well was added with $50 \mu \mathrm{L}$ prepared standards with different concentrations. The 96 well plate was incubated for $20 \mathrm{~min}$ at $37^{\circ} \mathrm{C}$. Then each well was added with $50 \mu \mathrm{L}$ reaction mix. Finally, the absorbance was measured on a microplate reader at $\mathrm{OD}=570 \mathrm{~nm}$ in kinetic mode at $37^{\circ} \mathrm{C}$. The arginase activity was calculated based on absorbance values and standard curve.

\section{Cell Culture and Calcium Oxalate Crystal Adhesion Assay}

$\mathrm{HK}-2$ cells were cultured in DMEM/F-12 supplemented with $10 \%$ foetal bovine serum at $37^{\circ} \mathrm{C}, 5 \% \mathrm{CO} 2$ and saturated humidity. The cells were cultured in six-well culture plates and exposed to sodium oxalate $(0.75 \mathrm{mM})$ and/or arginine $(0.86,1.72$, 4.31, 8.61, 17.22, and $43.05 \mathrm{mM}$ ) for $24 \mathrm{~h}$ (Salama et al., 2019; Rattazzi et al., 2020). After that, the medium was changed to culture medium containing $40 \mu \mathrm{g} / \mathrm{mL} \mathrm{CaOx}$ crystals for $10 \mathrm{~min}$ in a cell culture incubator. Then, the cells were washed with PBS thoroughly and examined under a bright field microscope (magnification, $\times 100$; CARL ZEISS, Heidenheim, Germany). We selected five different fields to calculate the number of adhesive crystals.

\section{Statistics}

Data are shown as the mean and standard deviation and were analysed by ANOVA and Student's $t$-test. $P$-values $<0.05$ were regarded as significantly different. GraphPad Prism (version 8) and R (version 3.6.1) were used for statistical analyses and to draw graphs.

\section{RESULTS}

\section{Lactiplantibacillus plantarum N-1 Reduced Renal Calcium Oxalate Crystals}

To explore the effect of $\mathrm{N}-1$ on renal $\mathrm{CaOx}$ crystal formation, we provided model rats with $\mathrm{N}-1$. After 4 weeks, HE staining showed that $1 \%$ EG successfully induced $\mathrm{CaOx}$ crystals in the kidney, which was also confirmed by VK staining. Supplementation with N-1 significantly reduced renal crystals (Figure 1A). The HE staining score and VK staining value (area) all increased significantly in the model group and decreased significantly in the N-1-supplemented group $(P<0.05)$ (Figures 1B,C). The 


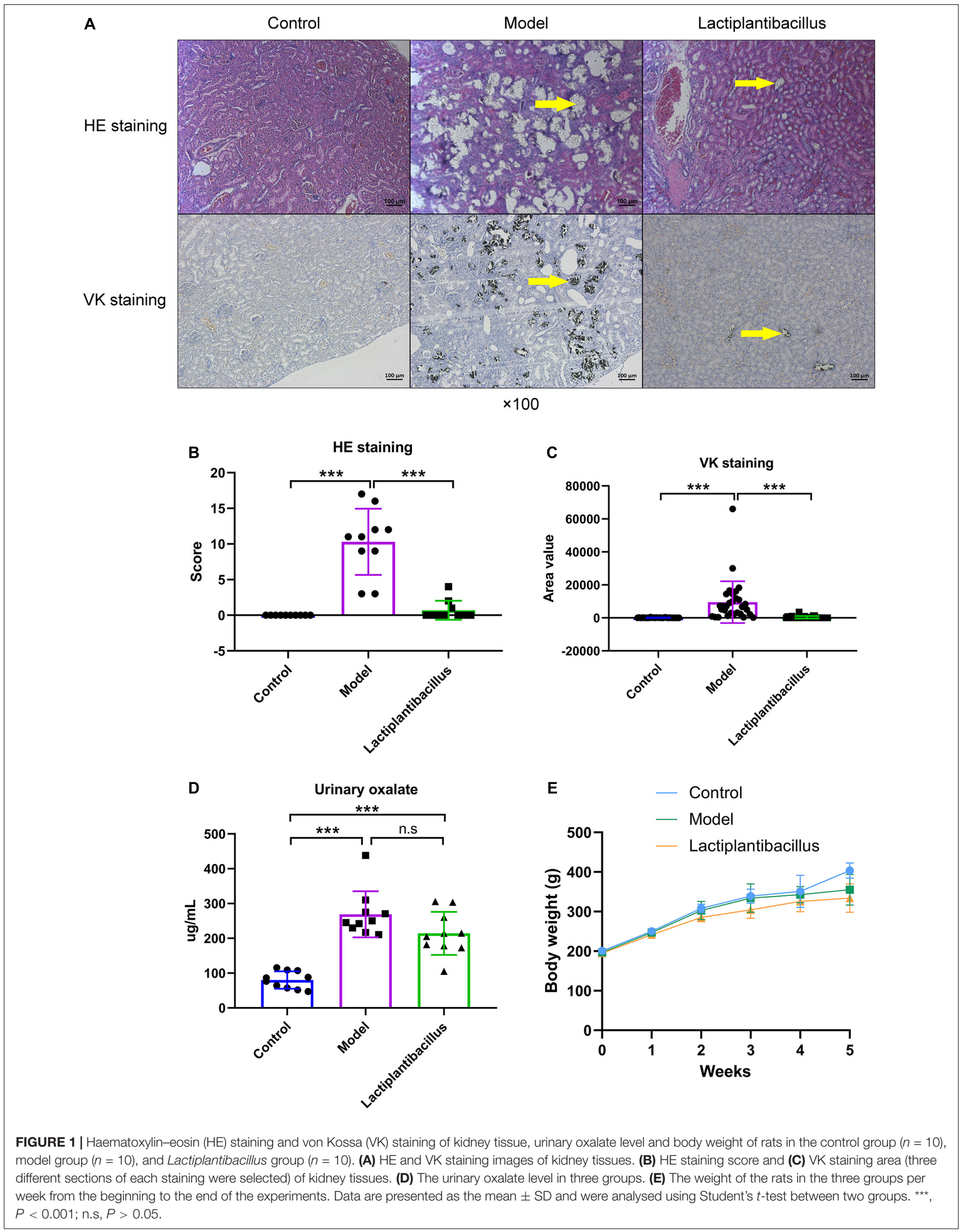


level of urinary oxalate was higher in the model rats than in the controls $(P<0.05)$. However, the administration of $\mathrm{N}-1$ did not affect urinary oxalate excretion $(P>0.05)$ (Figure 1D). The volume of $24 \mathrm{~h}$ urine was not statistically different between three groups $(P>0.05)$ (Supplementary Figure 1). The weights of all the rats increased steadily from the beginning to the end of the experiment. After 4 weeks, the weight of the model rats was lower than that of the control rats $(P<0.05)$, and they did not change after the administration of N-1 $(P>0.05)$ (Figure 1E).

\section{Lactiplantibacillus plantarum N-1 Reversed the Beta Diversity of the Gut Microbiota in the lleum}

To explore whether N-1 reduced renal crystals by regulating the gut microbiota, we next examined the gut microbiota. We found that the coverage indices were $>0.99$ in all groups at the OTU level, indicating that the sequencing depth was sufficient in all samples. The alpha diversity of the gut microbiota in the ileum, caecum, and colon was not different between the control, model, and N-1-supplemented groups (Supplementary Table 2).

The beta diversity of the gut microbiota was also evaluated by principal coordinate analysis (PCoA) using the BrayCurtis index. In the ileum, PCoA showed significant separation between the control and model rats $(P=0.019$, ADONIS). The beta diversity of the gut microbiota was different between the model group and the Lactiplantibacillus group $(P=0.045$, ADONIS $)$. The difference in the beta diversity between the control and N-1-supplemented groups decreased $(P=0.190$, ADONIS) (Figure 2A). In the caecum and colon, although the beta diversity was also different between the control and model groups, it did not change in the $\mathrm{N}$-1-supplemented group compared with the model group $(P>0.050$, ADONIS) (Supplementary Figures 2A,B). This evidence indicated that $\mathrm{N}-1$ could significantly affect the gut microbiota, especially in the ileum.

\section{Lactiplantibacillus plantarum N-1 Changed the Gut Microbiota}

At the phylum level, Firmicutes was the main component (7997\%). At the genus level, Lactobacillus was the most common bacterium. The relative abundance of Lactobacillus decreased from $65-83 \%$ in the ileum to $16-36 \%$ in the caecum and to $18-$ $46 \%$ in the colon. In the ileum, the percentage of Lactobacillus decreased from $83 \%$ in the control group to $65 \%$ in the model group and then increased to $75 \%$ in the $\mathrm{N}$-1-supplemented group (Figure 2B). In the caecum and colon, its percentage decreased in the model and N-1-supplemented groups compared with the control group, without any difference between the model and $\mathrm{N}$-1-supplemented groups (Supplementary Figures 2C,D). This evidence again suggested that $\mathrm{N}-1$ significantly regulated the gut microbiota in the ileum but not in the caecum or colon.

At the genus level, we used linear discriminant analysis effect size (LEfSe) to explore the different bacteria between the control, model, and N-1-supplemented groups. In the ileum, Staphylococcus, Corynebacterium 1, Jeotgalicoccus, Psychrobacter, and Aerococcus were more abundant in the

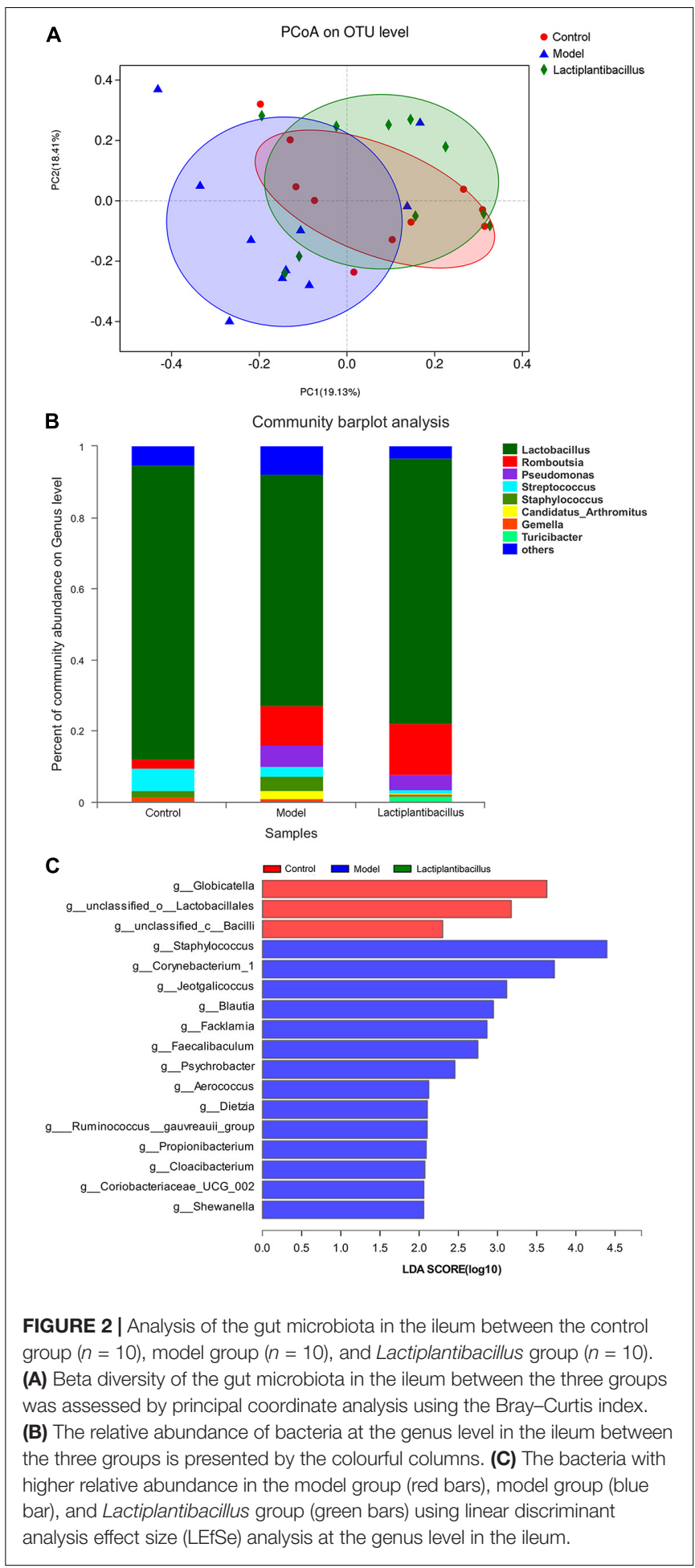

model group (Figure 2C). In contrast, the relative abundances of Lactobacillus and Eubacterium ventriosum were higher in the control group, and those of Ruminococcaceae UCG 007 and Rikenellaceae RC9 were higher in the N-1-supplemented group in the caecum (Supplementary Figure 3A) and colon (Supplementary Figure 3B). 


\section{Lactiplantibacillus plantarum $\mathrm{N}-1$ Regulated Metabolic Enzymes and Amino Acid Metabolism in the Gut}

Apart from bacteria, we also used phylogenetic investigation of communities by reconstruction of unobserved states (PICRUSt2) to predict the metabolic enzymes of the gut microbiota and analysed their differences between the three groups. Using LEfSe, we found that the levels of 19 enzymes were higher in the ileum model group (such as arginase). In addition, the levels of 12 enzymes were increased in the ileum after the administration of N-1 (such as L-serine ammonia-lyase). However, there were fewer significantly different enzymes between the model and N-1supplemented groups in the caecum and colon than in the ileum (Table 1).

To verify whether arginase and its metabolic substrate were involved in renal crystal formation, we examined the activity of arginase and found that it was lower in the ileal contents in the $\mathrm{N}$-1-supplemented group than in the model group (Figure 3A). We also examined the levels of different amino acids in the ileal contents (Supplementary Table 3) and blood (Supplementary Table 4). The level of arginine in the ileal contents was higher in the control group $(P=0.040)$ and N-1-supplemented group $(P=0.029)$ than in the model group (Figure 3B). The blood arginine level was also lower in the model group $(P=0.010)$ than in the control and N-1 groups $(P=0.021)$ (Figure 3C). The ornithine and citrulline levels in the ileal contents and blood was not significantly different between three groups (Supplementary Figure 4). In addition, the level of arginine in the ileal contents (Figure 3D) or blood (Figure 3E) was positively correlated with the bacteria at greater abundance in the control group and was negatively correlated with the bacteria at greater abundance in the model group.

\section{Arginine Ameliorated Calcium Oxalate Crystal-Renal Cell Adhesion}

To explore whether arginine could affect crystal adhesion of renal epithelial cells in vitro, we added $\mathrm{CaOx}$ crystals and arginine to the cell culture medium. The results showed that there was no adhesion of $\mathrm{CaOx}$ crystals to renal epithelial HK-2 cells when cells were treated with was arginine alone. The crystals adhesion increased after the exposure to $0.75 \mathrm{mM}$ oxalate, and reduced when cells were exposed to $0.75 \mathrm{mM}$ oxalate $+1.72 \mathrm{mM}$ or higher arginine $(P=0.023)$ (Figure 4).

\section{DISCUSSION}

Our previous study showed that $L$. plantarum $\mathrm{N}-1$ was resistant to acid and bile salt and, compared to other strains, had the highest survival rates of 98.0 and $99.1 \%$ under the conditions of pH 3.5 and $0.1 \%$ bile salt, respectively. This strain has the ability

TABLE 1| Higher predicted enzyme levels involved in the metabolic pathways of the gut microbiota in the ileum, caecum, and colon of the model and Lactiplantibacillus groups.

\begin{tabular}{|c|c|c|c|c|}
\hline & \multicolumn{2}{|r|}{ Model } & \multicolumn{2}{|r|}{ Lactiplantibacillus } \\
\hline & EC number & Name & EC number & Name \\
\hline \multirow[t]{19}{*}{ lleum } & f.3.1.21.3 & Type I site-specific deoxyribonuclease & f.4.3.1.17 & L-Serine ammonia-lyase \\
\hline & f.3.6.3.44 & Xenobiotic-transporting ATPase & f. 4.1 .2 .4 & Deoxyribose-phosphate aldolase \\
\hline & f.2.7.4.1 & Polyphosphate kinase & f.3.5.1.2 & Glutaminase \\
\hline & f.1.6.99.3 & NADH dehydrogenase & f.3.4.21.89 & Signal peptidase I \\
\hline & f.2.7.2.4 & Aspartate kinase & f.4.4.1.16 & Selenocysteine lyase \\
\hline & f.5.3.1.6 & Ribose-5-phosphate isomerase & f.5.1.3.2 & UDP-glucose 4-epimerase \\
\hline & f.2.7.7.9 & UTP-glucose-1-phosphate uridylyltransferase & f.2.8.3.19 & CoA:oxalate CoA-transferase \\
\hline & f.2.4.2.52 & Triphosphoribosyl-dephospho-CoA synthase & f.4.2.1.126 & $N$-acetylmuramic acid 6-phosphate etherase \\
\hline & f.4.1.1.20 & Diaminopimelate decarboxylase & f.2.1.1.163 & Demethylmenaquinone methyltransferase \\
\hline & f.1.2.1.11 & Aspartate-semialdehyde dehydrogenase & f.2.1.1.201 & 2-Methoxy-6-polyprenyl-1,4-benzoquinol methylase \\
\hline & f.3.5.4.26 & Diaminohydroxyphosphoribosylaminopyrimidine deaminase & f.3.4.21.102 & C-terminal processing peptidase \\
\hline & f.1.1.1.193 & 5-Amino-6-(5-phosphoribosylamino)uracil reductase & f.2.7.1.95 & Kanamycin kinase \\
\hline & f.2.3.1.81 & Aminoglycoside $N\left(3^{\prime}\right)$-acetyltransferase & & \\
\hline & f. $4.1 \cdot 3.42$ & (4S)-4-hydroxy-2-oxoglutarate aldolase & & \\
\hline & f.4.1.2.14 & 2-Dehydro-3-deoxy-phosphogluconate aldolase & & \\
\hline & f.5.1.1.7 & Diaminopimelate epimerase & & \\
\hline & f.4.1.2.17 & L-Fuculose-phosphate aldolase & & \\
\hline & f.2.7.1.45 & 2-Dehydro-3-deoxygluconokinase & & \\
\hline & f.3.5.3.1 & Arginase & & \\
\hline \multirow[t]{3}{*}{ Caecum } & f.1.3.5.4 & Fumarate reductase (quinol) & f.3.4.16.4 & Serine-type D-Ala-D-Ala carboxypeptidase \\
\hline & & & f.2.7.11.1 & Non-specific serine/threonine protein kinase \\
\hline & & & f.2.7.1.45 & 2-Dehydro-3-deoxygluconokinase \\
\hline \multirow[t]{3}{*}{ Colon } & f.2.7.6.5 & GTP diphosphokinase & f.2.7.7.13 & Mannose-1-phosphate guanylyltransferase \\
\hline & f.5.4.99.9 & UDP-galactopyranose mutase & & \\
\hline & f.1.1.3.15 & (S)-2-hydroxy-acid oxidase & & \\
\hline
\end{tabular}




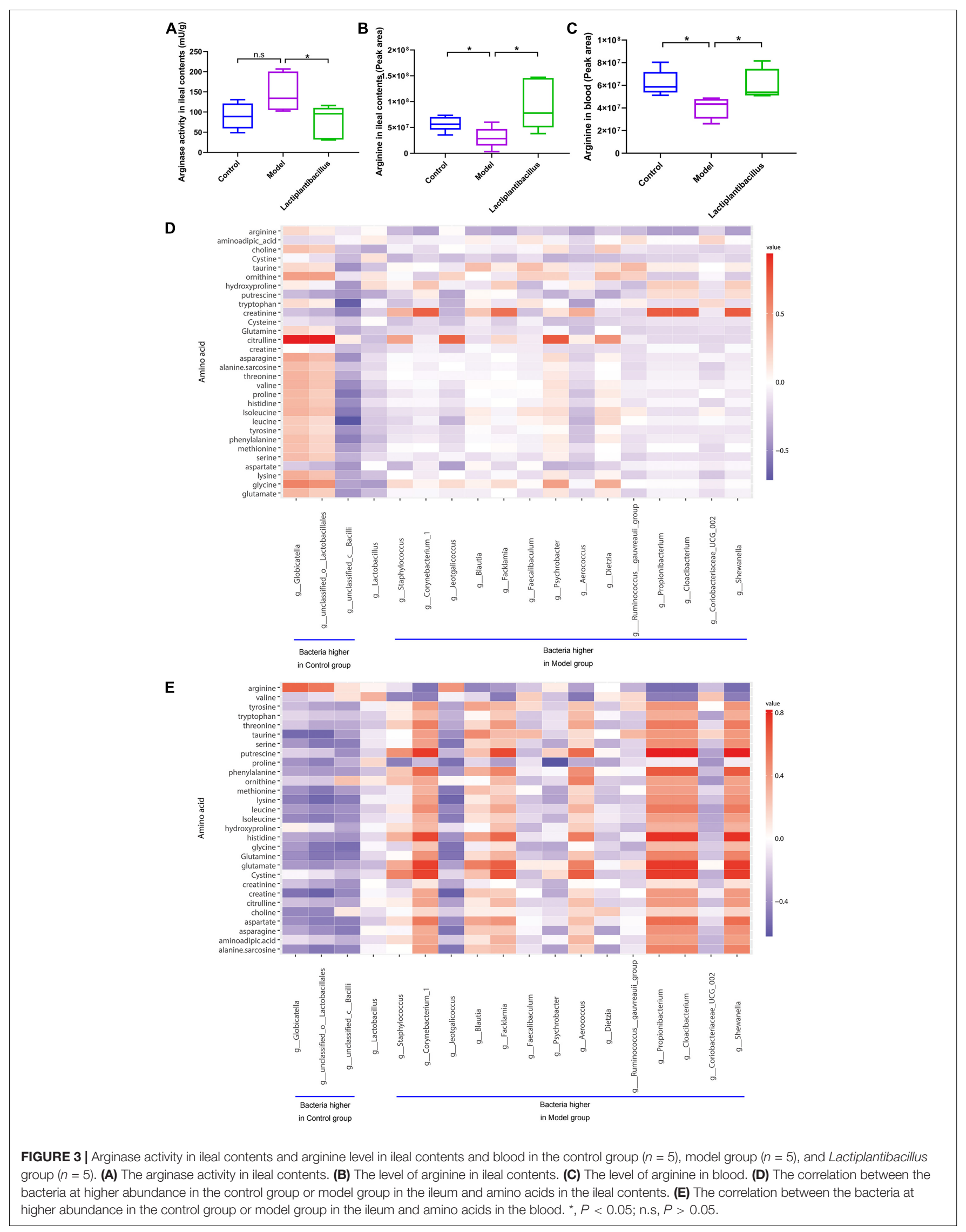



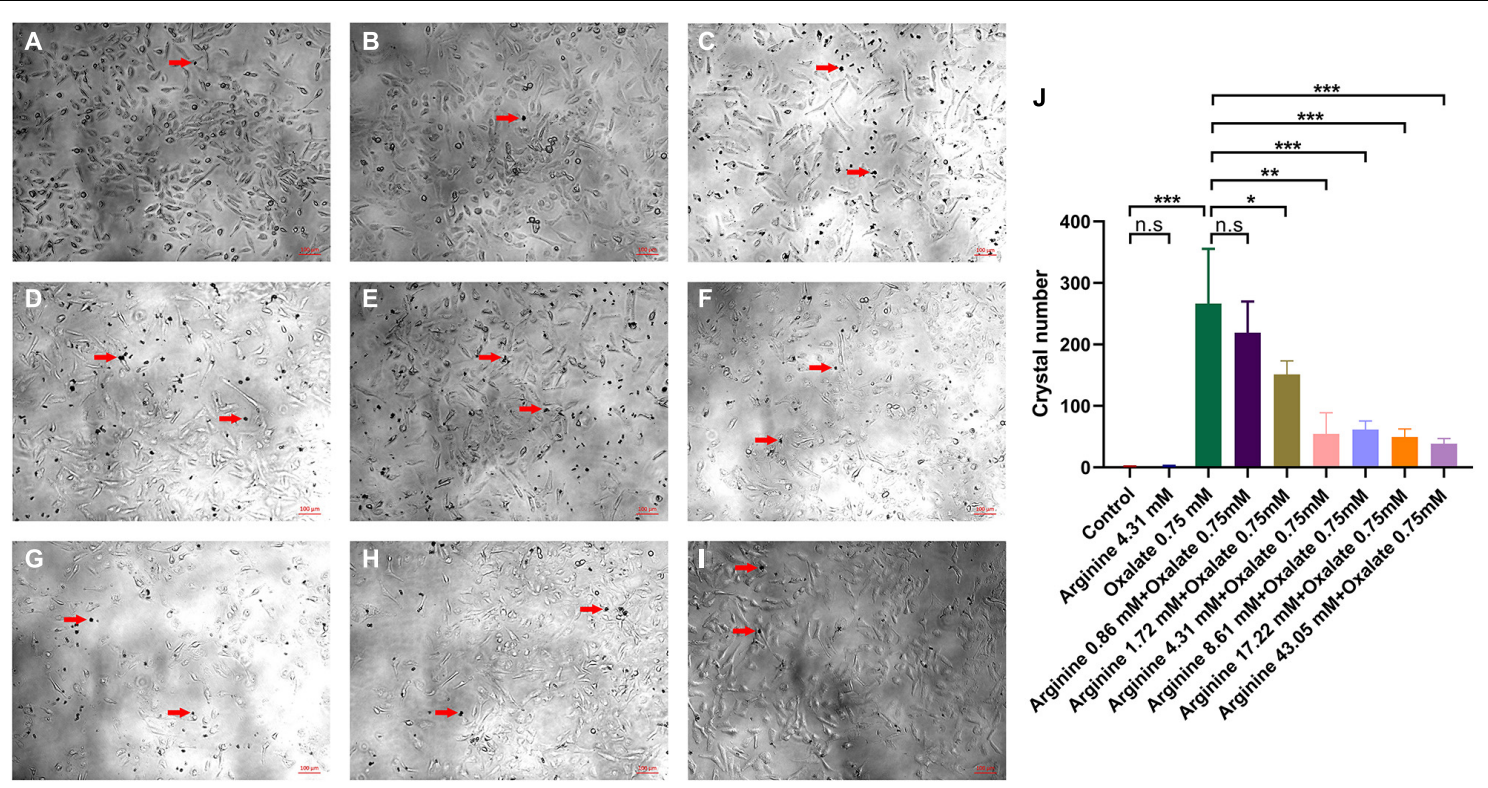

FIGURE 4 | The effect of sodium oxalate and arginine on the adhesion of $\mathrm{CaOx}$ crystals to renal epithelial HK-2 cells. CaOx crystal adhesion to renal epithelial HK-2 cells after exposure to (A) normal saline, (B) $4.31 \mathrm{mM}$ arginine, (C) $0.75 \mathrm{mM}$ sodium oxalate, (D) $0.86 \mathrm{mM}$ arginine $+0.75 \mathrm{mM}$ sodium oxalate, (E) $1.72 \mathrm{mM}$ arginine + $0.75 \mathrm{mM}$ sodium oxalate, (F) $4.31 \mathrm{mM}$ arginine $+0.75 \mathrm{mM}$ sodium oxalate, (G) $8.61 \mathrm{mM}$ arginine $+0.75 \mathrm{mM}$ sodium oxalate, (H) $17.22 \mathrm{mM}$ arginine $+0.75 \mathrm{mM}$ sodium oxalate, (I) $43.05 \mathrm{mM}$ arginine $+0.75 \mathrm{mM}$ sodium oxalate $(\times 100)$. The red arrow refers to the CaOx crystals. (J) Quantitative data for CaOx crystals adhering to the cells mentioned above. ${ }^{\star}, P<0.05 ;{ }^{* \star}, P<0.01 ;{ }^{* * \star}, P<0.001 ;$ n.s, $P>0.05$.

to adhere to intestines and antibacterial activity. In addition, the culture supernatants of $\mathrm{N}-1$ could inhibit the growth of Escherichia coli (Liu et al., 2017). All these features guaranteed the survival of $L$. plantarum $\mathrm{N}-1$ in the intestinal tract.

In a normal healthy person, $2-10 \%$ oxalate in the food is absorbed into blood, while the remaining part is degraded by the gut microbiota. In addition, some dietary precursors (such as glyoxylate and hydroxyproline) are also metabolised to oxalate as a terminal metabolite production in the liver (Sadaf et al., 2017). The oxalate is excreted through kidney and the intestine. Thus, the gut microbiota may play a key role in regulating the oxalate metabolism. It was reported that Lacticaseibacillus casei, capable of degrading oxalate in vitro, reduced urinary oxalate excretion and renal crystals in rats (Kwak et al., 2006). However, this study did not explore the underlying mechanisms. Later, some studies found that $L$. plantarum recombined with the oxalate decarboxylase gene could also reduce urinary levels (Sasikumar et al., 2014; Paul et al., 2018). However, these artificial bacteria may not be safe for humans. Some clinical studies also found that probiotics containing Lactobacillus could decrease urinary oxalate levels in idiopathic hyperoxaluria or renal stone patients, but the decline was not significantly different between probiotictreated patients and placebo-treated patients (Campieri et al., 2001; Lieske et al., 2005; Goldfarb et al., 2007; Siener et al., 2013). Interestingly, our study found that $L$. plantarum could reduce renal crystals without decreasing urinary oxalate excretion. Murphy et al. (2009) also reported that not all Lactobacillus could degrade oxalate. Thus, there may be some other mechanisms in addition to oxalate metabolism by which L. plantarum $\mathrm{N}-1$ in our study reduced renal crystal deposition.
Several studies have reported that the gut microbiota is different between renal $\mathrm{CaOx}$ stone patients and healthy controls, which suggests that the whole gut microbiota, instead of a single kind of bacterium, is important in the formation of renal stones. Thus, we further examined the composition of the gut microbiota in the ileum, caecum, and colon between the three groups. We found that Lactobacillus was the predominant bacterium in the gut, especially in the ileum. We also found that the beta diversity of the gut microbiota and the percentage of Lactobacillus in the $\mathrm{N}$-1-supplemented group were more similar to those in the control group, which indicated that $\mathrm{N}-1$ could reverse the gut microbiota of model rats to relatively normal conditions. However, this phenomenon was not observed in the caecum or colon. The most likely reason may be that the environment in the ileum was more suitable for Lactobacillus.

We also found that Lactobacillus was the main genus of the gut microbiota. Its relative abundance was much higher in the colon of the control group, which suggested that the decrease in Lactobacillus was associated with renal stones. Our study also verified that supplementation with L. plantarum N1 could reduce renal crystal formation. In addition, short-chain fatty acid (SCFA)-producing bacteria such as Eubacterium and Ruminococcaceae have been found in lower abundances in the gut microbiota of model groups (Wei and Tao, 2018; Xie et al., 2019). It was reported that SCFAs have anti-inflammatory functions (Koh et al., 2016). However, Staphylococcus, Corynebacterium 1, Jeotgalicoccus, Psychrobacter, and Aerococcus were higher in the gut microbiota of the model group of this study, and these genera are associated with acute or chronic inflammatory diseases, including inflammatory bowel diseases, necrotising 
lymphadenitis, and diabetes mellitus (El Mouzan et al., 2018; Salem et al., 2019; Xu et al., 2019; Zhou et al., 2019; Chen et al., 2020). This evidence suggested that N-1 could directly or indirectly ameliorate the dysbiosis of the gut microbiota and decrease inflammatory levels in various organs and tissues (such as kidneys) by regulating the gut microbiome, followed by less damage to renal cells and fewer crystals (Khan, 2014).

In this study, we predicted that a higher level of arginase would be found in the ileal microbiota of the model group than in the $\mathrm{N}$-1-supplemented group. The predictive results also showed that the arginase level in caecal or colonic contents was not significantly different between model group and $\mathrm{N}$ 1-supplemented group. Meanwhile, the relative abundance of Lactobacillus was higher in the ileal contents than in the caecal and colonic contents. Thus, we hypothesised that the supplemented Lactobacillus mainly regulated the arginase activity and arginine level in the ileal contents. Further examinations verified that the arginase activity was lower in the ileal contents of N-1-supplemented rats than in the model rats. Arginine levels were higher in the ileal contents and blood of $\mathrm{N}$-1-supplemented rats than in the model rats. Ornithine and citrulline are the key arginine metabolites, where arginine is metabolised to ornithine through arginase. Thus, we also compared the ornithine and citrulline levels in the ileal contents and blood. Though they were not significantly different between three groups, there was a decrease trend of ornithine level in the ileal contents after the supplementation of $\mathrm{N}-1$.

Additionally, an in vitro study also showed that arginine could reduce $\mathrm{CaOx}$ crystal adhesion to renal cells. Kandhare et al. (2015) and Pragasam et al. (2005) reported that the administration of L-arginine could increase urinary citrate and reduce free radicals and oxalate in the kidney in rats with renal crystals. Kizivat et al. (2017) also found that L-arginine could reduce oxidative stress in kidney epithelial cells exposed to oxalate in vitro. Arginine could also suppress the inflammatory response and apoptosis of intestinal epithelial cells (Zhang et al., 2020a,b). All this evidence suggests that L. plantarum N-1 may reduce renal crystals by regulating the metabolism of gut microbiota and increasing the level of arginine in the gut.

The present study was performed only on Sprague-Dawley rats. The effect of $\mathrm{N}-1$ on preventing renal $\mathrm{CaOx}$ stones in humans needs further research. However, our study also had several advantages. First, N-1, isolated from traditional cheese in China, is not pathogenic to humans and is easily obtained. Second, compared with the study performed by Kwak et al. (2006), our study is the first to explore the change in gut microbiota after the administration of L. plantarum and to discuss its effect on preventing renal $\mathrm{CaOx}$ stones by decreasing arginase levels in the gut microbiota. These results will promote further study of the mechanisms underlying renal $\mathrm{CaOx}$ stone formation.

\section{CONCLUSION}

Our findings highlighted the important role of L. plantarum $\mathrm{N}-1$ in reducing renal $\mathrm{CaOx}$ crystals by regulating arginine metabolism in the gut microbiota. Probiotics containing
L. plantarum N-1 may be potential therapies for preventing renal $\mathrm{CaOx}$ stones.

\section{DATA AVAILABILITY STATEMENT}

The data presented in the study are deposited in the NCBI repository, accession number: PRJNA759335.

\section{ETHICS STATEMENT}

The animal study was reviewed and approved by West China Hospital of Sichuan University Medical Research Ethics Committee.

\section{AUTHOR CONTRIBUTIONS}

YL, XJ, QS, and KW conceived and designed the experiments. YL, LT, YM, LC, and YC performed the experiments, analysed the data, and wrote the manuscript. ZJ, XJ, HL, QS, and KW reviewed and edited the manuscript. QS and KW provided guidance for the experiments. All authors contributed to the article and approved the submitted version.

\section{FUNDING}

This research was funded by the National Natural Science Foundation of China (81970602 and 81770703), the Foundation of Science and Technology Department of Sichuan Province (2018SZ0118 and 2019YFS0281), the 1.3.5 Project for Disciplines of Excellence, West China Hospital, Sichuan University (ZYJC18015), the National Key Research and Development Projects (SQ2019YFE010495), and the PostDoctor Research Project, West China Hospital, Sichuan University (2019HXBH087).

\section{ACKNOWLEDGMENTS}

We specially thank staffs participating in experimental animal experiments and molecular biology experiments, including YC, Xiaofang Xu, Zude Chen, Lina Gong, Jianzhong Ai, Banghua Liao, Qing $\mathrm{He}$, and Guo Chen. We thank Guang Yang, Xijing Yang, and Xiaoting Chen for administrative support at Animal Experiment Center of West China Hospital, Sichuan University. We thank Li Li, Fei Chen, and Chunjuan Bao from the Institute of Clinical Pathology, West China Hospital of Sichuan University for helping with processing the histological sections and staining.

\section{SUPPLEMENTARY MATERIAL}

The Supplementary Material for this article can be found online at: https://www.frontiersin.org/articles/10.3389/fmicb.2021. 743097/full\#supplementary-material 


\section{REFERENCES}

Al-Wahsh, I., Wu, Y., and Liebman, M. (2012). Acute probiotic ingestion reduces gastrointestinal oxalate absorption in healthy subjects. Urol. Res. 40, 191-196. doi: 10.1007/s00240-011-0421-7

Antonelli, J. A., Maalouf, N. M., Pearle, M. S., and Lotan, Y. (2014). Use of the National Health and Nutrition Examination Survey to calculate the impact of obesity and diabetes on cost and prevalence of urolithiasis in 2030. Eur. Urol. 66, 724-729. doi: 10.1016/j.eururo.2014.06.036

Campieri, C., Campieri, M., Bertuzzi, V., Swennen, E., Matteuzzi, D., Stefoni, S., et al. (2001). Reduction of oxaluria after an oral course of lactic acid bacteria at high concentration. Kidney Int. 60, 1097-1105. doi: 10.1046/j.1523-1755.2001. 0600031097.x

Chen, M., Xiao, D., Liu, W., Song, Y., Zou, B., Li, L., et al. (2020). Intake of Ganoderma lucidum polysaccharides reverses the disturbed gut microbiota and metabolism in type 2 diabetic rats. Int. J. Biol. Macromol. 155, 890-902. doi: 10.1016/j.ijbiomac.2019.11.047

El Mouzan, M. I., Winter, H. S., Assiri, A. A., Korolev, K. S., Al Sarkhy, A. A., Dowd, S. E., et al. (2018). Microbiota profile in new-onset pediatric Crohn's disease: data from a non-Western population. Gut Pathog. 10:49.

Goldfarb, D. S., Modersitzki, F., and Asplin, J. R. (2007). A randomized, controlled trial of lactic acid bacteria for idiopathic hyperoxaluria. Clin. J. Am. Soc. Nephrol. 2, 745-749. doi: 10.2215/cjn.00600207

Hatch, M., Gjymishka, A., Salido, E. C., Allison, M. J., and Freel, R. W. (2011). Enteric oxalate elimination is induced and oxalate is normalized in a mouse model of primary hyperoxaluria following intestinal colonization with Oxalobacter. Am. J. Physiol. Gastrointest. Liver Physiol. 300, G461-G469.

Hoppe, B. (2012). An update on primary hyperoxaluria. Nat. Rev. Nephrol. 8, 467-475. doi: 10.1038/nrneph.2012.113

Hoppe, B., Pellikka, P. A., Dehmel, B., Banos, A., Lindner, E., and Herberg, U. (2021). Effects of Oxalobacter formigenes in subjects with primary hyperoxaluria Type 1 and end-stage renal disease: a Phase II study. Nephrol. Dial. Transplant. 36, 1464-1473. doi: 10.1093/ndt/gfaa135

Kandhare, A. D., Patil, M. V. K. and Bodhankar, S. L. (2015). L-Arginine attenuates the ethylene glycol induced urolithiasis in ininephrectomized hypertensive rats: role of KIM-1, NGAL, and NOs. Ren. Fail. 37, 709-721. doi: 10.3109/0886022x. 2015.1011967

Khan, S. R. (2014). Reactive oxygen species, inflammation and calcium oxalate nephrolithiasis. Transl. Androl. Urol. 3, 256-276.

Kizivat, T., Smoliæ, M., Mariæ, I., Tolušiæ Levak, M., Smoliæ, R., Biliæ Eurèiæ, I., et al. (2017). Antioxidant pre-treatment reduces the toxic effects of oxalate on renal epithelial cells in a cell culture model of urolithiasis. Int. J. Environ. Res. Public Health 14:109. doi: 10.3390/ijerph14010109

Knoll, T., Schubert, A. B., Fahlenkamp, D., Leusmann, D. B., Wendt-Nordahl, G., and Schubert, G. (2011). Urolithiasis through the ages: data on more than 200,000 urinary stone analyses. J. Urol. 185, 1304-1311. doi: 10.1016/j.juro. 2010.11.073

Koh, A., De Vadder, F., Kovatcheva-Datchary, P., and Bäckhed, F. (2016). From dietary fiber to host physiology: short-chain fatty acids as key bacterial metabolites. Cell 165, 1332-1345. doi: 10.1016/j.cell.2016.05.041

Kwak, C., Jeong, B. C., Ku, J. H., Kim, H. H., Lee, J. J., Huh, C. S., et al. (2006). Prevention of nephrolithiasis by Lactobacillus in stone-forming rats: a preliminary study. Urol. Res. 34, 265-270. doi: 10.1007/s00240-006-0 054-4

Lieske, J. C., Goldfarb, D. S., De Simone, C., and Regnier, C. (2005). Use of a probiotic to decrease enteric hyperoxaluria. Kidney Int. 68, 1244-1249. doi: 10.1111/j.1523-1755.2005.00520.x

Liu, R., Tian, Y., Geng, Q., Shi, J., Zhang, Y., and Sun, Q. (2017). Isolation, identification and functional properties of lactic acid bacteria derived from traditional cheese in Daocheng. Sichuan. China Meas. Test 43, 58-62.

Liu, Y., Chen, Y., Liao, B., Luo, D., Wang, K., Li, H., et al. (2018). Epidemiology of urolithiasis in Asia. Asian J. Urol. 5, 205-214. doi: 10.1016/j.ajur.2018.08.007

Lopes, R., Balbino, K. P., Jorge, M. P., Ribeiro, A. Q., Martino, H. S. D., and Alfenas, R. C. G. (2018). Modulation of intestinal microbiota, control of nitrogen products and inflammation by pre/probiotics in chronic kidney disease: a systematic review. Nutr. Hospital. 35, 722-730.

Milliner, D., Hoppe, B., and Groothoff, J. (2018). A randomised Phase II/III study to evaluate the efficacy and safety of orally administered Oxalobacter formigenes to treat primary hyperoxaluria. Urolithiasis 46, 313-323. doi: 10.1007/s00240017-0998-6

Minisola, S., Gianotti, L., Bhadada, S., and Silverberg, S. J. (2018). Classical complications of primary hyperparathyroidism. Best Pract. Res. Clin. Endocrinol. Metab. 32, 791-803. doi: 10.1016/j.beem.2018.09.001

Murphy, C., Murphy, S., O’Brien, F., O’Donoghue, M., Boileau, T., Sunvold, G., et al. (2009). Metabolic activity of probiotics-oxalate degradation. Vet. Microbiol. 136, 100-107. doi: 10.1016/j.vetmic.2008.10.005

Okombo, J., and Liebman, M. (2010). Probiotic-induced reduction of gastrointestinal oxalate absorption in healthy subjects. Urol. Res. 38, 169-178. doi: 10.1007/s00240-010-0262-9

Paul, E., Albert, A., Ponnusamy, S., Mishra, S. R., Vignesh, A. G., Sivakumar, S. M., et al. (2018). Designer probiotic Lactobacillus plantarum expressing oxalate decarboxylase developed using group II intron degrades intestinal oxalate in hyperoxaluric rats. Microbiol. Res. 215, 65-75. doi: 10.1016/j.micres.2018.06. 009

Pragasam, V., Kalaiselvi, P., Sumitra, K., Srinivasan, S., and Varalakshmi, P. (2005). Counteraction of oxalate induced nitrosative stress by supplementation of 1-arginine, a potent antilithic agent. Clin. Chim. Acta 354, 159-166. doi: 10. 1016/j.cccn.2004.11.029

Rattazzi, M., Donato, M., Bertacco, E., Millioni, R., Franchin, C., Mortarino, C., et al. (2020). 1-Arginine prevents inflammatory and pro-calcific differentiation of interstitial aortic valve cells. Atherosclerosis 298, 27-35. doi: 10.1016/j. atherosclerosis.2020.02.024

Sadaf, H., Raza, S. I., and Hassan, S. W. (2017). Role of gut microbiota against calcium oxalate. Microb. Pathog. 109, 287-291. doi: 10.1016/j.micpath.2017.06. 009

Salama, A. A. K., Duque, M., Wang, L., Shahzad, K., Olivera, M., and Loor, J. J. (2019). Enhanced supply of methionine or arginine alters mechanistic target of rapamycin signaling proteins, messenger RNA, and microRNA abundance in heat-stressed bovine mammary epithelial cells in vitro. J. Dairy Sci. 102, 2469-2480. doi: 10.3168/jds.2018-15219

Salem, F., Kindt, N., Marchesi, J. R., Netter, P., Lopez, A., Kokten, T., et al. (2019). Gut microbiome in chronic rheumatic and inflammatory bowel diseases: Similarities and differences. U. Eur. Gastroenterol. J. 7, 1008-1032. doi: 10.1177/ 2050640619867555

Sasikumar, P., Gomathi, S., Anbazhagan, K., Abhishek, A., Paul, E., Vasudevan, V., et al. (2014). Recombinant Lactobacillus plantarum expressing and secreting heterologous oxalate decarboxylase prevents renal calcium oxalate stone deposition in experimental rats. J. Biomed. Sci. 21:86.

Sidhu, H., Allison, M. J., Chow, J. M., Clark, A., and Peck, A. B. (2001). Rapid reversal of hyperoxaluria in a rat model after probiotic administration of Oxalobacter formigenes. J. Urol. 166, 1487-1491. doi: 10.1097/00005392200110000-00078

Siener, R., Bade, D. J., Hesse, A., and Hoppe, B. (2013). Dietary hyperoxaluria is not reduced by treatment with lactic acid bacteria. J. Transl. Med. 11:306. doi: 10.1186/1479-5876-11-306

Sorokin, I., Mamoulakis, C., Miyazawa, K., Rodgers, A., Talati, J., and Lotan, Y. (2017). Epidemiology of stone disease across the world. World J. Urol. 35 1301-1320. doi: 10.1007/s00345-017-2008-6

Tundo, G., Khaleel, S., and Pais, V. M. Jr. (2018). Gender equivalence in the prevalence of nephrolithiasis among adults younger than 50 Years in the United States. J. Urol. 200, 1273-1277. doi: 10.1016/j.juro.2018.07.048

Wei, X., and Tao, J. (2018). Xiexin Tang improves the symptom of type 2 diabetic rats by modulation of the gut microbiota. Sci. Rep. 8:3685.

Wu, W., Yang, D., Tiselius, H. G., Ou, L., Mai, Z., Chen, K., et al. (2015). Collection and storage of urine specimens for measurement of urolithiasis risk factors. Urology 85, 299-303. doi: 10.1016/j.urology.2014.10.030

Xiang, S., Zhou, J., Li, J., Wang, Q., Zhang, Q., Zhao, Z., et al. (2015). Antilithic effects of extracts from different polarity fractions of Desmodium styracifolium on experimentally induced urolithiasis in rats. Urolithiasis 43, 433-439. doi: 10.1007/s00240-015-0795-Z

Xie, J., Liu, Y., Chen, B., Zhang, G., Ou, S., Luo, J., et al. (2019). Ganoderma lucidum polysaccharide improves rat DSS-induced colitis by altering cecal microbiota and gene expression of colonic epithelial cells. Food Nutr. Res. 12:63.

Xu, J., Huang, G., Nagy, T., and Guo, T. L. (2019). Bisphenol A alteration of type 1 diabetes in non-obese diabetic (n.d.) female mice is dependent on window of exposure. Arch. Toxicol. 93, 1083-1093. doi: 10.1007/s00204-019-02419-4 
Zeng, G., Mai, Z., Xia, S., Wang, Z., Zhang, K., Wang, L., et al. (2017). Prevalence of kidney stones in China: an ultrasonography based cross-sectional study. BJU Int. 120, 109-116. doi: 10.1111/bju.13828

Zhang, H., Ma, Y., Wang, M., Elsabagh, M., Loor, J. J., and Wang, H. (2020a). Dietary supplementation of l-arginine and $\mathrm{N}$-carbamylglutamate enhances duodenal barrier and mitochondrial functions and suppresses duodenal inflammation and mitophagy in suckling lambs suffering from intrauterine-growth-restriction. Food Funct. 11, 4456-4470. doi: 10.1039/d0fo0 0019a

Zhang, H., Zhao, F., Peng, A., Guo, S., Wang, M., Elsabagh, M., et al. (2020b). 1-Arginine inhibits apoptosis of ovine intestinal epithelial cells through the 1-arginine-nitric oxide pathway. J. Nutr. 150, 2051-2060. doi: 10.1093/jn/ nxaa133

Zhou, Z., Li, H., Tian, S., Yi, W., Zhou, Y., Yang, H., et al. (2019). Critical roles of NLRP3 inflammasome in IL-1 $\beta$ secretion induced by Corynebacterium pseudotuberculosis in vitro. Mol. Immunol. 116, 11-17. doi: 10.1016/j.molimm. 2019.09.016
Conflict of Interest: The authors declare that the research was conducted in the absence of any commercial or financial relationships that could be construed as a potential conflict of interest.

Publisher's Note: All claims expressed in this article are solely those of the authors and do not necessarily represent those of their affiliated organizations, or those of the publisher, the editors and the reviewers. Any product that may be evaluated in this article, or claim that may be made by its manufacturer, is not guaranteed or endorsed by the publisher.

Copyright (C) 2021 Liu, Jin, Tian, Jian, Ma, Cheng, Cui, Li, Sun and Wang. This is an open-access article distributed under the terms of the Creative Commons Attribution License (CC BY). The use, distribution or reproduction in other forums is permitted, provided the original author(s) and the copyright owner(s) are credited and that the original publication in this journal is cited, in accordance with accepted academic practice. No use, distribution or reproduction is permitted which does not comply with these terms. 